\title{
A YOUNG GIRL WITH REPEATED EPISODES OF PNEUMONIA AND INTERMITTENT DIARRHOEA- THE PRIMARY IMMUNODEFICIENCY SYNDROME
}

\author{
ABDULLAH-AL-MAMUN ${ }^{1}$, SAMIM HASAN ${ }^{1}$, SYED AHMED ABDULLAH ${ }^{1}$, AHMED RIYAD HOSSAIN ${ }^{2}$, SAFIUL \\ ALAM $^{3}$, ROBED AMIN ${ }^{4}$, MA KAHHAR ${ }^{5}$
}

\begin{abstract}
:
A description is given of a case of a young girl who presented with repeated episodes of pneumonia since childhood. In addition, she had episodes of intermittent diarrhoea. Her investigative work-up revealed a deficiency of serum immunoglobulins. The profile was suggestive of common variable immunodeficiency. Because of a relative rarity of this disease, it is often misdiagnosed earlier. Late diagnosis and delayed institution of immunoglobulin replacement therapy results in increased morbidity with a wide variety of organ-specific complications and increased mortality.
\end{abstract}

\section{Introduction:}

The primary immunodeficiency syndromes are a rare group of disorders that can present at an age for which delay in diagnosis remains common. Persons with hypogamma globulinaemia are more likely to get infections and patient suffer from recurrent infections of various body systems, more so of the respiratory tract. Replacement therapy with immunoglobulins in primary immunodefiency increases life expectancy and reduces infection frequency and severity ${ }^{1}$. We present a case of a young female who developed repeated chest infections, recurrent diarrhoea and arthritis since early childhood.

\section{Case report:}

A 23 years old female presented with pain and swelling of multiple joints for last 3 months. Pain first appeared at right side of the pelvis. About one month later both wrists, all metacarpophalangeal, shoulders, elbows and knees were successively involved. Pain was constant, moderate to severe in nature and sometimes became so severe that patient could not move the joints and she had to take analgesics regularly. Pain was associated with swelling of the joints but not associated with morning stiffness and redness. Pain spared back, chest, neck and feet. For this pain she consulted a rheumatologist who diagnosed her as a case of ankylosing spondylitis and prescribed Salphasalazine. But the patient could not tolerate the drug. She had also history of getting intra-articular steroid injection. The patient also mentioned that for last 20 days she developed fever and burning sensation during micturition and loose motion. Her father also stated that since childhood she was growth retarded and suffered recurrent fever, respiratory tract infection, loose motion, burning sensation during micturition and oral sore and for this she got medication for multiple times. On query, patient had a history of taking anti-tubercular drugs for sputum negative pulmonary tuberculosis. She also underwent lobectomy of left lung for bronchiectasis 4 months back. (Fig 1)

1. Honorary Medical Officer, Department of Medicine, Dhaka Medical College

2. Assistant Registrar, Department of Medicine, Dhaka Medical College

3. Indoor Medical Officer, Department of Medicine, Dhaka Medical College

4. Assistant Professor of Medicine, Dhaka Medical College

5. Professor of Medicine, Dhaka Medical College, Department of Medicine Unit V, Dhaka Medical College Hospital, Dhaka.

Bangladesh J Medicine 2011; 22 : 24-26 


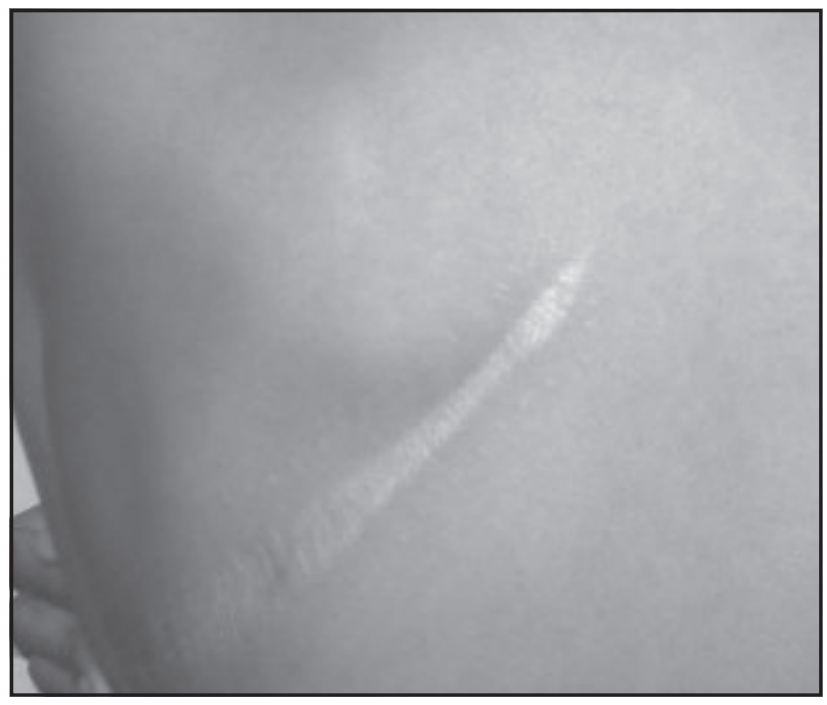

Fig.-1: The scar ofleft sided lobectomy due to bronchiectasis

Patient is asthmatic and it was diagnosed as cough variant asthma with positive bronchoprovocation test 4 months back and for this she was on medication for last 3 months. On examination, patient was emaciated; pulse-80/min ; blood pressure-70/50 mm of $\mathrm{Hg}$; temp- $99^{\circ} \mathrm{F}$; Anaemia -moderate; clubbing present; no palpable lymphadenopathy \& thyroid gland was not enlarged. Nutritional status: BMI-9.4kg/m2; mid upper arm circumference- $15 \mathrm{~cm}$. Examination of lower extrimities \& pelvis- bilateral sacroiliac joint tenderness; tenderness over both knee joints but no restrictive movement; tenderness over lower lumbar spine but no gibbus; Schober's test was positive; SLR negative. Examination of upper extrimities-wasting of the small muscles of the hand \& dorsal guttering, ulnar deviation of hands; tenderness over both wrists, metacarpophalangeal joints, elbow \& shoulder joints and there was restricted movement of the wrist \& shoulders, no tenderness over proximal \& distal interphalangeal joints.(Fig.2)

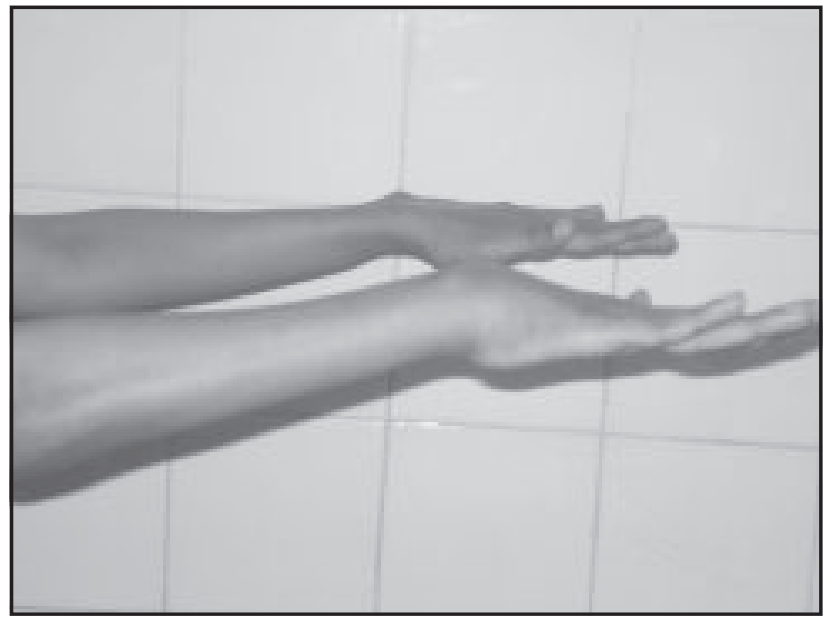

Fig 2: Wasting of hand muscles and ulnr deviation
Investigations revealed- $\mathrm{CBC}: \mathrm{Hb}-7.7 \mathrm{gm} \%, \mathrm{ESR}-45$ $\mathrm{mm}$ in $1^{\text {st }}$ hour, WBC count-10.68 thousand/mm3, Neutrophil-76\%, Lymphocyte-18\% ,Hematocrit26.60\%, MCV-78.50 fl. CRP-1.72 mg/L(0-3 mg/L). RA test -negative,Anti CCP antibody $<1$ unit/ml. ANAnegative. HLA-B27-positive.Serum ferritin-827 ng/ $\mathrm{ml}(15-200 \mathrm{ng} / \mathrm{ml})$. Blood C/S- no growth of organisms. Urine R/M/E-pus cell: 10-12/hpf, epithelial cells:24/hpf, RBC- nil, Albumin-absent. Urine C/S-no growth of organisms. Urine AFB-not found. Stool R/ $\mathrm{M} / \mathrm{E}$ : mucous ++ , vegetable cells ++. Stool culture no growth of organism. $X$ ray Pelvis A/P view: no significant skeletal abnormality. $\mathrm{X}$ ray of lumbosacral spine $\mathrm{B} / \mathrm{V}$ : no significant skeletal abnormality. $\mathrm{X}$ ray chest P/A view: old fracture or expansile bony lesion at the posterior aspect of left $6^{\text {th }}$ rib. $\mathrm{X}$ ray hand $\mathrm{B} / \mathrm{V}$ : Left- suggestive of inflammatory joint disease; Right -mild subluxation of fifth metacarpophalangeal joint.(Fig.-3).

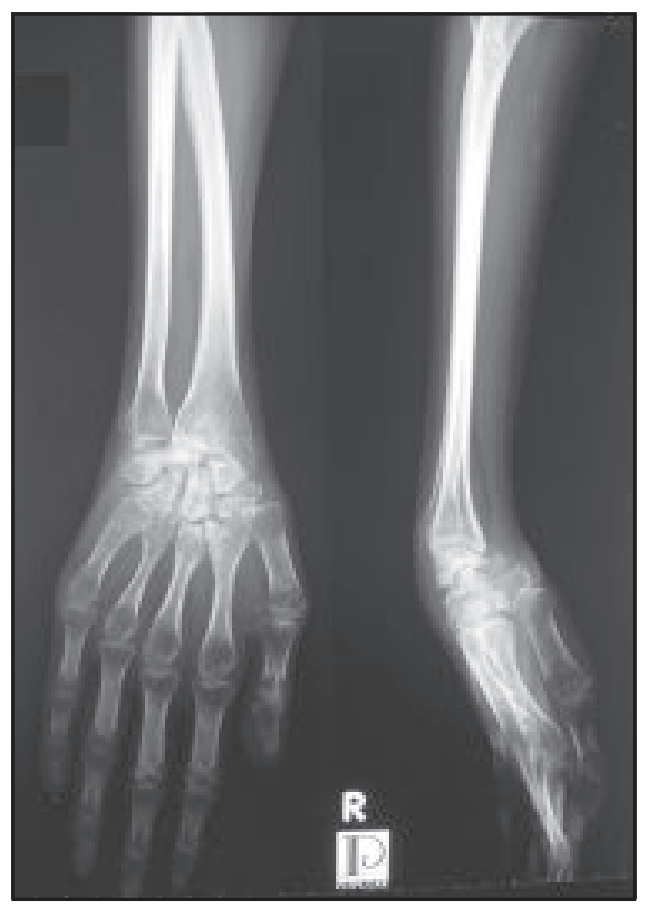

Fig.-3: X ray hands and forearm revealed subluxation of fifth metacarpophalangeal

USG of whole abdomen- Echogenic liver parenchyma. Liver Function Test-normal. FBG-4.6 mmol/L. S. creatinine- $1.08 \mathrm{mg} / \mathrm{dl}$. S electrolytes: sodium$135 \mathrm{mmol} / \mathrm{L}$, potassium-4.7 mmol/L,chloride-98 $\mathrm{mmol} / \mathrm{L}, \mathrm{CO} 2-24 \mathrm{mmol} / \mathrm{L}$. Serum immunoglobulin study- Ig G-2.56 gm/L(7-16), IgM-0.17 gm/L(0.4-2.3), Ig A-0.23 gm/L(0.70-4.00) CT scan of chest- triangular density areas containing air densities are seen along medial aspects of left lower lobe, impression of small 
chronic pneumonia with bronchiectatic changes suggestive of chronic TB. Pneumonia CT guided FNAC of lower left lung- smear shows scanty cellular material containing a few polymorphs, lymphocytes $\&$ pulmonary macrophages in the background of blood. No malignant or granuloma is seen; suggestive of inflammatory lesion. Histopathology : section of the lung shows multiple epitheloid granuloma with caseation necrosis with bronchiectatic changes; no focal alveolar haemorrage-suggestive of bronchiectatic changes with PTB. Sputum for AFB (three consecutive morning samples)-negative. Echocardiographynormal. After evaluation clinically and investigations she was diagnosed as a case of Primary Immunodeficiency Syndrome

\section{Discussion:}

Immunoglobulin deficiency disorders are an important though uncommon cause of recurrent infections, which are not often suspected by treating physicians. The classical feature of such disorders is an increased susceptibility to infections and immunodeficiency should be suspected in any patient who presents with recurrent or persistent infections ${ }^{2}$. Defects in antibody synthesis may be primary or secondary and may be quantitative or qualitative. The major cause of primary antibody deficiency includes common variable immunodeficiency, selective IgA deficiency, infantile sex linked hypogammaglobulinaemia (Brutons disease), transient hypogammaglo nulinaemia of infancy, IgG subclass deficiency etc. Secondary causes of antibody deficiency could be either due to decreased production(malnutrition, lymphoproliferative disease, drugs) or increased losses(nephrotic syndrome, protein losing enteropathy, burns) ${ }^{3}$

Patient with defects in humoral immunity have recurrent or chronic sinopulmonary infection, meningitis, and bacteremia, most commonly caused by pyogenic bacteria such as Haemophilus influenzae, Streptococcus pneumoniae, and Staphylococcus aureus. Abnormalities of $\mathrm{T}$ cell-mediated immunity predispose to disseminated virus infections, particularly with latent viruses such as Herpes simplex. Varicella zoster and Cytomegalovirus. In addition, patient so affected almost invariably develop mucocutaneous candidiasis and frequently acquire systemic fungal infections ${ }^{4}$.

Recurrent infections usually begin at about one year of age because maternally transferred antibody affords some passive protection till that time. Growth retardation is a common symptoms. Delay in diagnosis is frequent and is overlooked for many years before a diagnosis is made and treatment started A survey in England showed a delay in diagnosis in half of all children and virtually all adults. The length of ranged from 2-27 years in adults and 1 to 5 years in children $^{5}$. IgA deficiency of common variable immunodeficiency(CVID) represent polar ends of a clinical spectrum due to the same underlying gene
defect(s).Over a period of years, IgA-deficient patient may progress to the panhypogammaglobulinaemia phenotype characteristic of CVID, and vice versa. Patient with isolated IgA deficiency may present with an increased number of respiratory infections that may lead to bronchiectasis. Chronic diarrhoeal diseases also occur. It is also associated with arthritis and systemic lupus erythematosus. Patient with CVID may also present with signs and symptoms suggestive of lymphoid malignancy, including fever, weight loss, anemia, thrombocytopenia, splenomegaly, generalized lymphadenopathy, and lymphocytosis. Once suspected, lab diagnosis is simple and measurement of serum immunoglobulins will provide the diagnosis. If the level of immunoglobulins is normal, testing for antibody subclasses or functional antibody may be indicated. Serum complement levels and a normal lymphocyte count will serve as screening test to rule out co-existing deficiency disorders ${ }^{6}$.

Pulmonary abnormalities develop in most patients with primary hypogammaglobulinemia. A new finding is that silent and asymptomatic progression of pulmonary changes may occur in patients despite an adequate immunoglobulin replacement therapy. Highresolution computed tomography is the method of choice in monitoring pulmonary changes 7 . The administration of intravenous immunoglobulin in adequate doses is an essential part of the prevention and treatment of all these complications ${ }^{4}$. We treated our patient with intravenous immunoglobulin(dose $400 \mathrm{mg} / \mathrm{kg}$ ) once. Immediately after giving immunoglobulin she felt better including improvement of joint pain. She had improvement on general well being, appetite and was afebrile during hospital stay. There was no adverse event seen during or after administration of immunoglobulin. Patient was advised for follow up.

\section{References:}

1. Philip Wood. The value of early diagnosis and treatment of patients with primary antibody deficiencies;: Pharmaceuticals Policy and Law. Volume 2008; 10:147-157.

2. Malaviya AN, Rajagopal P, Taneja RL. Pattern of primary immunodeficiencies seen in India. J AOI .1977; 25: 465-74.

3. Chapel H, Haeney M. Essentials of Clinical Immunology; $2^{\text {nd }}$ edition. Oxford: Blackwell Scientific Publications; 1988; 68-108.

4. Harrison's principles of Internal Medicine; $17^{\text {th }}$ edition; Primary Immune Deficiency Diseases: 20542060 pages.

5. Blore J, Haeney MR. Primary antibody deficiency and diagnostic delay. Br Med J 1989; 298:516-7.

6. Datta U, Kumar L, Metha S, et al. Primary immunodeficiency defects seen in PGI: One year study. JAPI 1984; 32: 701-4.

7. Cunningham-Rundles $\mathrm{C}$, Bodian C. Common variable immunodeficiency: clinical and immunological features of 248 patients. Clin Immunol. Jul 1999; 92(1):34-48. 